\title{
On linear systems of $\mathbb{P}^{3}$ with nine base points
}

\author{
Maria Chiara Brambilla ${ }^{1}$. Olivia Dumitrescu ${ }^{2}$. \\ Elisa Postinghel ${ }^{3}$
}

Received: 27 December 2014 / Accepted: 21 August 2015 / Published online: 4 September 2015

(C) Fondazione Annali di Matematica Pura ed Applicata and Springer-Verlag Berlin Heidelberg 2015

\begin{abstract}
We study special linear systems of surfaces of $\mathbb{P}^{3}$ interpolating nine points in general position having a quadric as fixed component. By performing degenerations in the blown-up space, we interpret the quadric obstruction in terms of linear obstructions for a quasi-homogeneous class. By degeneration, we also prove a Nagata type result for the blown-up projective plane in points that implies a base locus lemma for the quadric. As an application, we establish Laface-Ugaglia Conjecture for linear systems with multiplicities bounded by 8 and for homogeneous linear systems with multiplicity $m$ and degree up to $2 m+1$.
\end{abstract}

Keywords Fat points · Degeneration techniques · Laface-Ugaglia Conjecture · Base locus · Quadric surface

Mathematics Subject Classification Primary: 14C20 · Secondary: 14J70, 14 J26

Maria Chiara Brambilla is partially supported by MIUR and INDAM. Olivia Dumitrescu is a member of the Simion Stoilow Institute of Mathematics of the Romanian Academy. Elisa Postinghel is supported by the Research Foundation-Flanders (FWO).

$凶$ Elisa Postinghel

elisa.postinghel@wis.kuleuven.be

Maria Chiara Brambilla

brambilla@dipmat.univpm.it

Olivia Dumitrescu

dumitrescu@math.uni-hannover.de

1 Università Politecnica delle Marche, via Brecce Bianche, 60131 Ancona, Italy

2 Institut für Algebraische Geometrie GRK 1463, Welfengarten 1, 30167 Hannover, Germany

3 Department of Mathematics, KU Leuven, Celestijnenlaan 200B, 3001 Heverlee, Belgium 


\section{Introduction}

The theory of linear systems is a classical object of study which is related to secant varieties, polynomial interpolation and to several interesting recently discovered applications. Even if linear systems have been studied for more than a century, basic questions, such as the dimensionality problem, are still open in general.

We denote by $\mathcal{L}=\mathcal{L}_{n, d}\left(m_{1}, \ldots, m_{s}\right)$ the linear system of hypersurfaces of degree $d$ in $\mathbb{P}^{n}$ interpolating $s$ points in general position with multiplicities at least, respectively, $m_{1}, \ldots, m_{s}$. A linear system is said to be non-special if it has the (affine) expected dimension, which is $\operatorname{edim}(\mathcal{L})=\max (\operatorname{vdim}(\mathcal{L}), 0)$, where the $(\operatorname{affine})$ virtual dimension $\operatorname{vdim}(\mathcal{L})$ is defined as

$$
\operatorname{vdim}(\mathcal{L})=\left(\begin{array}{c}
n+d \\
n
\end{array}\right)-\sum_{i=1}^{s}\left(\begin{array}{c}
n+m_{i}-1 \\
n
\end{array}\right) .
$$

Special linear systems are those that have dimension strictly higher than the expected one and the speciality of the system is the difference

$$
\operatorname{dim}(\mathcal{L})-\operatorname{edim}(\mathcal{L})=\mathrm{h}^{1}(\mathcal{L}) \geq 0 .
$$

In general, computing the dimension of the linear systems is a challenging task. In order to classify the special linear systems, one has to understand first what are the obstructions, namely what are the varieties that, whenever contained with multiplicity in the base locus of $\mathcal{L}$, generate speciality. In $[3,4]$, these obstructions are named special effect varieties.

The well-known Alexander-Hirschowitz theorem ([1], see also [7,30]), which concerns the case of linear systems with double points in $\mathbb{P}^{n}$, provides a list of special systems where the special effect varieties are linear cycles (when $d=2$ ), a rational normal curve (when $d=3$ ) or a quadric hypersurface (when $d=4$ ).

For higher multiplicities, the planar case has been deeply investigated by many authors. For $n=2$, the famous Segre-Harbourne-Gimigliano-Hirschowitz Conjecture states that the obstructions are given by $(-1)$-curves $[19,23,24,32]$ (see also [11,12,14]).

For the higher-dimensional case, in $[5,18]$ the authors extensively studied the linear special effect varieties in $\mathbb{P}^{n}$. In particular, knowing the exact contribution to the speciality of any multiple linear cycle contained in the base locus allows to introduce the notion of linear expected dimension (see [5, Definition 3.2]). We say that a system is linearly non-special whenever its dimension is equal to the linear expected dimension. This happens exactly when the only special effect varieties are linear cycles.

The authors devote the paper [6] to the investigation of linear systems in $\mathbb{P}^{n}$ with $n+3$ base points having nonlinear obstructions. More precisely, the rational normal curve through the points is a special effect curve.

It is a well-known fact that Cremona reduced linear systems of $\mathbb{P}^{3}$ do not contain rational normal curves in their base locus. Laface and Ugaglia conjectured [27] that for a Cremona reduced linear system, the only special effect varieties are lines and quadric surfaces determined by nine points. The Conjecture of Laface-Ugaglia is known to be true if the number of points is less or equal than eight [15], and when the maximal multiplicity of the points is five [2].

In this paper, we study linear systems in $\mathbb{P}^{3}$ with at least nine fat points in general position for which the quadric hypersurface through nine of the base points, namely the fixed surface $Q:=\mathcal{L}_{3,2}\left(1^{9}\right)$, is a special effect variety. 
The first step is to prove a base locus lemma for quadric surfaces. Even obtaining a weak base locus lemma is not obvious. In fact, such results can be obtained as a consequence of Nagata type results, i.e. theorems which prove emptiness, for linear systems in $\mathbb{P}^{2}$ with ten points. In Sect. 4, we establish a base locus lemma for the quadric surface through nine points (see Theorem 4.1) for a particular class of linear systems in $\mathbb{P}^{3}$. In order to prove this result, we study the emptiness of linear systems with ten points in $\mathbb{P}^{2}$, via a suitable degeneration technique inspired by $[10,13]$.

The next step is to classify the special linear systems whose special effect varieties are quadrics. In particular, we focus on the case of (Cremona reduced) linear systems with nine points in $\mathbb{P}^{3}$, which is the first case where the speciality is not due only to linear obstructions.

Our goal is to understand precisely how much the quadric surface in the base locus contributes to the speciality of the system. Unlike the linear case, to give a formula which computes exactly the contribution to the speciality seems difficult in general (see Remark 5.5 for more details).

Hence, we focus first on some particular classes of linear systems that are the homogeneous and the quasi-homogeneous ones.

The first case we study is given by the quasi-homogeneous linear systems $\mathcal{L}_{3,2 m}\left(m^{8}, a\right)$, for $1 \leq a \leq m$. This class of systems behaves surprisingly well; indeed, we are able to find an easy formula which relates the speciality with the multiplicity of the quadric in the base locus, see Theorem 3.1. The proof of this result is based on a degeneration argument, which allows to reduce the "mysterious" contribution of the quadric to the sum of two contributions given by linear special effect varieties in the degenerated systems.

We recall that in the literature various degeneration arguments have been used to prove non-speciality results of linear systems in the plane $[11,12,17]$ and in higher dimension $[26,30]$.

In the case of degree $2 m+1$, the relation between the speciality and the quadric becomes less clear even in the homogeneous case. However in Theorem 4.12, we classify all the special homogeneous linear systems with nine points of multiplicity $m$ and degree $2 m+1$. In order to prove this result, we apply the emptiness results mentioned above and proved in Sect. 4.1.

In the last section, as an application of Theorems 3.1 and 4.12, we show that the LafaceUgaglia Conjecture holds for linear systems of any degree and nine points of multiplicity at most 8. In order to complete this proof as well as the proof of Theorem 4.12, some of the computations are made by means of the computer algebra system Macaulay2 [22].

We want to point out finally that the quadric hypersurfaces are sporadic special effect varieties. Indeed, it is expected (see e.g. the Fröberg-Iarrobino Conjecture for homogeneous linear systems, [9, Conjecture 4.8]) that they give contribution to the speciality of a linear system only in $\mathbb{P}^{3}$ and $\mathbb{P}^{4}$. We think that the understanding of the case of linear systems in $\mathbb{P}^{3}$ with nine points is the initial step in order to investigate the special systems obstructed by a quadric.

This article is organized as follows. In Sect. 2, we give a brief description of the tools that we will use to prove our results.

In Sect. 3, we classify the case $\mathcal{L}_{3,2 m}\left(m^{8}, a\right)$ and we give a geometric interpretation, via degenerations, of the quadric as special effect surface.

In Sect. 4, we completely classify the case $\mathcal{L}_{3,2 m+1}\left(m^{9}\right)$; the main results are Theorem 4.1 and Theorem 4.2.

In Sect. 5, we prove that Laface-Ugaglia Conjecture holds for linear systems with nine base points of multiplicities $m_{i} \leq 8$. 


\section{Preliminaries}

In this preliminary section, we collect general results and techniques that will be used throughout the paper. We point out that by dimension of a linear system $\mathcal{L}$, we mean the affine dimension $\operatorname{dim}(\mathcal{L})=\mathrm{h}^{0}(\mathcal{L})$, and not the projective dimension.

\subsection{Degenerations}

A natural approach to the dimensionality problem of linear systems is via degenerations. Degenerations allow to move the multiple base points of a linear system in special position and compute the dimension via a semi-continuity argument.

In $[11,12]$, Ciliberto and Miranda exploited a degeneration of the plane, originally proposed by Ran [31] to study higher multiplicity interpolation problems for planar linear systems with general multiple base points. This approach consists in degenerating the plane to a reducible surface, with two components intersecting along a line, and simultaneously degenerating the linear system to a limit linear system which is somewhat easier than the original one. In particular, this degeneration argument allows to use induction either on the degree or on the number of imposed multiple points. This method was generalized by the third author to the higher-dimensional cases of $\mathbb{P}^{n}[30]$ and of $\left(\mathbb{P}^{1}\right)^{n}$ with Laface [26].

Let $X \subseteq \mathbb{P}^{N}$ be a variety, let $\Delta$ be a complex disc with centre at the origin and let $\mathcal{X} \rightarrow \Delta$ be a one-dimensional embedded degeneration of $X$ to the union of two varieties $X^{1}, X^{2}$, i.e. a one-parameter family $\left\{X_{t}\right\}_{t \in \Delta}$ such that $X_{t} \cong X, t \neq 0$ and $X_{0}=X^{1} \cup X^{2}$. Let $\mathcal{L}_{t}:=\mathcal{L}$ be a line bundle on the general fibre.

A limit $\mathcal{L}_{0}$ of $\mathcal{L}_{t}$ is a line bundle on $X_{0}$ obtained as fibred product of a line bundle $\mathcal{L}^{1}$ on $X^{1}$ and a line bundle $\mathcal{L}^{2}$ on $X^{2}$ over the intersection of the restricted line bundles $\mathcal{L}^{1}{ }_{\mid Y}$ and $\mathcal{L}^{2}{ }_{\mid Y}$. This provides a recursive formula for the dimension of $\mathcal{L}_{0}$ in terms of the dimensions of the involved linear systems on the two components:

$$
\operatorname{dim}\left(\mathcal{L}_{0}\right)=\operatorname{dim}\left(\hat{\mathcal{L}}^{2}\right)+\operatorname{dim}\left(\hat{\mathcal{L}}^{1}\right)+\operatorname{dim}\left(\mathcal{L}^{1}{ }_{\mid Y} \cap \mathcal{L}^{2}{ }_{\mid Y}\right),
$$

where $\hat{\mathcal{L}}^{i}$ is the kernel of the restriction map $\mathcal{L}^{i} \rightarrow \mathcal{L}^{i}{ }_{\mid Y}, i=1,2$. Upper semi-continuity implies the inequality $\operatorname{dim}\left(\mathcal{L}_{t}\right) \leq \operatorname{dim}\left(\mathcal{L}_{0}\right)$.

\subsection{Linear systems on $Q \cong \mathbb{P}^{1} \times \mathbb{P}^{1}$}

In order to study the base locus of linear systems on $\mathbb{P}^{3}$ through nine general points, we want to understand their restrictions to the quadric surface $Q=\mathcal{L}_{3,2}\left(1^{9}\right) \cong \mathbb{P}^{1} \times \mathbb{P}^{1}$. The restriction of $\mathcal{L}=\mathcal{L}_{3, d}\left(m_{1}, \ldots, m_{9}\right)$ will be the linear series of curves of bidegree $(d, d)$ on $Q$ with nine multiple points in general position that we will denote as

$$
\mathcal{L}_{\mathbb{P}^{1} \times \mathbb{P}^{1},(d, d)}\left(m_{1}, \ldots, m_{9}\right) .
$$

Not very much is known about such linear systems: Giuffrida, Maggioni and Ragusa were among the first to study linear systems on a quadric surface in [20], see e.g. [21]. As far as we know, the only cases completely classified are those of double points [33] and triple points [25].

The following result allows to transform linear systems of given bidegree on the quadric $\mathbb{P}^{1} \times \mathbb{P}^{1}$ with multiple base points to linear systems on $\mathbb{P}^{2}$ with multiple base points, and vice versa, by means of cut-and-sew of polygons.

The image of $\mathbb{P}^{2}$ blown-up at two points via the embedding given by the linear system $\mathcal{L}_{2, d_{1}+d_{2}-m}\left(d_{1}-m, d_{2}-m\right)$ based at two torus-invariant points (e.g. two coordinate points) 

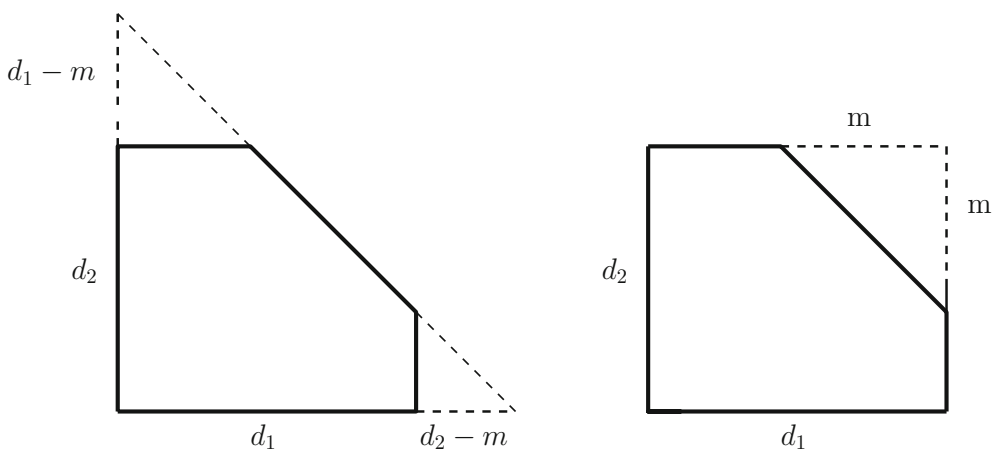

Fig. 1 Two equivalent polytopes

is a toric projective surface whose defining polytope is combinatorially equivalent to the pentagon obtained by the triangle $\left(d_{1}+d_{2}-m\right) \Delta$ by cutting two triangles $\left(d_{1}-m\right) \Delta$ and $\left(d_{2}-m\right) \Delta$ from two corners, where $\Delta$ is the two simplex of $\mathbb{R}^{2}$, see Fig. 1 on the left-hand side.

Notice that the same polytope can be obtained from the rectangle $\left[0, d_{1}\right] \times\left[0, d_{2}\right] \subset \mathbb{R}^{2}$ by cutting off the triangle $m \Delta$ from a corner. This interprets the above toric surface as the embedding of $\mathbb{P}^{1} \times \mathbb{P}^{1}$ via the linear system of curves of bidegree $\left(d_{1}, d_{2}\right)$ with a point of multiplicity $m$, that is $\mathcal{L}_{\mathbb{P}^{1} \times \mathbb{P}^{1},\left(d_{1}, d_{2}\right)}(m)$, see Fig. 1 on the right-hand side.

In other terms, this is the birational map that factors in the blow-up of $\mathbb{P}^{2}$ at two points and the blow-down of the (-1)-line joining them.

This proves the following result.

Lemma 2.1 If $m \leq d_{1}, d_{2}$, then the following equality holds

$\operatorname{dim}\left(\mathcal{L}_{\mathbb{P}^{1} \times \mathbb{P}^{1},\left(d_{1}, d_{2}\right)}\left(m, m_{1}, \ldots, m_{s}\right)\right)=\operatorname{dim}\left(\mathcal{L}_{2, d_{1}+d_{2}-m}\left(d_{1}-m, d_{2}-m, m_{1}, \ldots, m_{s}\right)\right)$.

Remark 2.2 In [8, Theorem 1.1], the authors show how to convert linear systems on products of projective spaces $\mathbb{P}^{n_{i}}$ interpolating multiple points into linear systems in the projective space $\mathbb{P}^{\sum n_{i}}$ interpolating multiple points and multiple linear subspaces, and back. We point out that the case $m=0$ in Lemma 2.1 falls into those equivalences, in the particular case of $\mathbb{P}^{1} \times \mathbb{P}^{1}$ and $\mathbb{P}^{2}$.

\subsection{Cremona transformations}

We recall that the standard Cremona transformation of $\mathbb{P}^{n}$ is the birational transformation defined by the following rational map:

$$
\mathrm{Cr}:\left(x_{0}: \cdots: x_{n}\right) \rightarrow\left(x_{0}^{-1}: \cdots: x_{n}^{-1}\right) .
$$

This map induces an action on the Picard group of the $n$-dimensional space blown-up at $s$ points. Let $\mathcal{L}=\mathcal{L}_{n, d}\left(m_{1}, \ldots, m_{s}\right)$ be a linear system based on $s$ points in general position; we can assume, without loss of generality, that the first $n+1$ points are the coordinate points. The Cremona action on $\mathcal{L}$ is described by the following rule (see, for example, [16,27]). Set

$$
c:=m_{1}+\cdots+m_{n+1}-(n-1) d,
$$

then

$$
\operatorname{Cr}(\mathcal{L})=\mathcal{L}_{n, d-c}\left(m_{1}-c, \ldots, m_{n+1}-c, m_{n+2}, \ldots, m_{s}\right)
$$


and

$$
\operatorname{dim}(\mathcal{L})=\operatorname{dim}(\operatorname{Cr}(\mathcal{L}))
$$

We will use this transformations in the cases $n=2,3$ to reduce the computation of the dimension of a linear system $\mathcal{L}$ to the computation of the dimension of its Cremona transform $\operatorname{Cr}(\mathcal{L})$ that has lower degree and multiplicities whenever $c>0$.

If $c \leq 0$, we will say that the linear system $\mathcal{L}$ is Cremona reduced.

\subsection{Computing with Macaulay2}

In this paper, we will need to perform some explicit computations in order to complete our classifications. In particular, the proofs of Proposition 4.9, Lemma 4.10, Lemma 5.7 and Theorem 5.8 are computer aided. We perform these computations by means of the computer algebra system Macaulay2. The procedure we use consists essentially in checking that several square matrices, randomly chosen, have maximal rank. We work over a field of characteristic 31991 and the proofs hold also in characteristic zero.

We use two scripts (one for linear systems in $\mathbb{P}^{2}$ and one in $\mathbb{P}^{3}$ ) available at this url http:// dipmat.univpm.it/ brambilla/NinePointsP3.html, which allow to compute the dimension and the speciality of a linear system with given degree and multiplicities.

\section{Quasi-homogeneous linear systems $\mathcal{L}_{3,2 m}\left(m^{8}, a\right)$}

In this section, we describe a class of special linear systems in $\mathbb{P}^{3}$ with nine base points for which the quadric surface $Q$ is the only special effect variety. We employ a double degeneration argument, similar to the one employed in [30] for linear systems with arbitrary general double points, that is based on the degeneration of the space described in Sect. 2.1. The linear system will degenerate into one that has only linear special effect varieties and that is therefore understood by the results in $[5,18]$.

Fix non-negative integers $a, m$. Consider the quasi-homogeneous linear system in $\mathbb{P}^{3}$

$$
\mathcal{L}(m, a):=\mathcal{L}_{3,2 m}\left(m^{8}, a\right) .
$$

The main result of this section is the following.

Theorem 3.1 If $1 \leq a \leq m$, the linear system (3.1) satisfies

$$
\begin{aligned}
& \operatorname{dim}(\mathcal{L}(m, a))=m-a+1, \\
& h^{1}(\mathcal{L}(m, a))=\left(\begin{array}{c}
a+1 \\
3
\end{array}\right)+\left(\begin{array}{l}
a \\
2
\end{array}\right) ;
\end{aligned}
$$

hence, it is special if and only if $2 \leq a \leq m$. Moreover, the only special effect variety for $\mathcal{L}(m, a)$ is the quadric through nine points which is contained in the base locus with exact multiplicity $a$.

It was proved already in [28, Section 5] that for $a=m$, the linear system (3.1) has one element, that is the $m$-multiple of the quadric through the nine points. This also implies that if $a>m$, the linear system (3.1) is empty. The case $a=0$ was proved to be non-special in [15]. So the remaining cases to explore are $1 \leq a \leq m-1$; for the sake of completeness, we include here the proof of the case $a=m$ as well. 
Theorem 3.1 shows that the linear system (3.1) is special with dimension being a linear function of $m$ and $a$. The only special effect variety is the quadric through the nine points which is contained with multiplicity $a$ in the base locus, and moreover, quite surprisingly, its contribution to the speciality, namely $h^{1}(\mathcal{L}(m, a))$, only depends on the multiplicity of containment of the quadric.

Remark 3.2 If we define $q(\mathcal{L}(m, a)):=\chi\left(\mathcal{L}(m, a)_{\mid Q}\right)$ to be the Euler characteristic of the restriction of $\mathcal{L}(m, a)$ to the quadric, (see (5.1) in Sect. 5), then one can easily check the following:

$$
q(\mathcal{L}(m, a))=1-\left(\begin{array}{c}
a+1 \\
2
\end{array}\right)<0 \text { iff } a \geq 2, \text { and } q(\mathcal{L})=0 \text { if } a=1 .
$$

This in particular shows that Theorem 3.1 has the following immediate consequence:

Corollary 3.3 Laface-Ugaglia Conjecture (see Conjecture 5.1 in Sect. 5) is true for any quasi-homogeneous linear system of the form (3.1).

\subsection{Degeneration of the blown-up $\mathbb{P}^{3}$ at 9 points}

In this section, we give a detailed description of the degeneration techniques that we will employ to prove Theorem 3.1.

\subsubsection{First degeneration}

Consider the trivial family $\mathcal{V}=\mathbb{P}^{3} \times \Delta \rightarrow \Delta$ with fibres $V_{t} \cong \mathbb{P}^{3}, t \in \Delta$. The blow-up of a point $p_{0} \in V_{0}$ produces a flat morphism $\mathcal{X}^{\prime} \rightarrow \Delta$ with general fibre $X_{t}^{\prime} \cong \mathbb{P}^{3}$ and central fibre $X_{0}^{\prime}=\mathbb{F} \cup \mathbb{P}$, where $\mathbb{F} \cong \mathrm{Bl}_{p_{0}} \mathbb{P}^{3}$ is the pullback of $V_{0}$ and $\mathbb{P} \cong \mathbb{P}^{3}$ is the exceptional divisor in the total space $\mathcal{X}^{\prime}$. The two components $\mathbb{F}$ and $\mathbb{P}$ meet transversally along a surface $Y \cong \mathbb{P}^{2}$ that, as a divisor, belongs to the exceptional class of $\mathbb{F}$ and to the hyperplane class of $\mathbb{P}$. More precisely, if $E_{0}:=\left.\mathbb{P}\right|_{\mathbb{F}}$ denotes the exceptional divisor of $p_{0} \in V_{0}, H^{\mathbb{F}}$ the hyperplane class of $\mathbb{F}$ and $H^{\mathbb{P}}$ that of $\mathbb{P}$, with $H^{\mathbb{P}} \sim E_{0}$, we have $\operatorname{Pic}(\mathbb{F})=\left\langle H^{\mathbb{F}}, E_{0}\right\rangle$ and $\operatorname{Pic}(\mathbb{P})=\left\langle H^{\mathbb{P}}\right\rangle$.

We choose seven general points on $\mathbb{F}$ and two points on $\mathbb{P}$, and we consider them as limits of nine general points in the general fibre $X_{t}^{\prime}$. More precisely, for $t \in \Delta$ let $\left\{p_{1}(t) \ldots, p_{9}(t)\right\}$ be a general collection of points and assume that $p_{1}(0), \ldots, p_{7}(0) \in \mathbb{F}$ while $p_{8}(0)$ and $p_{9}(0) \in \mathbb{P}$. Consider $\widetilde{\mathcal{X}}^{\prime}$ the blow-up of $\mathcal{X}^{\prime}$ along the horizontal curves $\left\{p_{i}(t)\right\}_{t \in \Delta}$, with exceptional divisors $\mathcal{E}_{i}, i=1, \ldots, 9$. Denote by $\widetilde{X}_{t}^{\prime}, t \in \Delta$ the fibres of the new family. Write also $E_{i}:=\left.\mathcal{E}_{i}\right|_{\tilde{X}_{t}^{\prime}}$, for $t \in \Delta, i=1, \ldots, 9$. The general fibre is $\widetilde{X}_{t}^{\prime} \cong \mathrm{Bl}_{p_{1}, \ldots, p_{9}}\left(\mathbb{P}^{3}\right)$, the blow-up of $\mathbb{P}^{3}$ at nine general points, so that $\operatorname{Pic}\left(\widetilde{X}_{t}\right)=\left\langle H, E_{1}, \ldots, E_{9}\right\rangle$. The central fibre is described by $\operatorname{Pic}(\mathbb{F})=\left\langle H^{\mathbb{F}}, E_{0}, E_{1}, \ldots, E_{7}\right\rangle$ and $\operatorname{Pic}(\mathbb{P})=\left\langle H^{\mathbb{P}}, E_{8}, E_{9}\right\rangle$, where by abuse of notation, $\mathbb{F}$ and $\mathbb{P}$ are also the pullbacks in $\tilde{\mathcal{X}}^{\prime}$ of the components of $X_{0}^{\prime}$.

\subsubsection{Second degeneration}

We further specialize the points by sending a point from each component of $X_{0}^{\prime}$ to the intersection. More precisely, consider the trivial family $\mathcal{X}^{\prime \prime}:=X_{0}^{\prime} \times \Delta^{\prime}$ and, on each fibre over $s \in \Delta^{\prime}$, take a collection of general points $\left\{p_{1}(s), \ldots, p_{7}(s)\right\} \subset \mathbb{F}$ and $\left\{p_{8}(s), p_{9}(s)\right\} \subset \mathbb{P}$ such that, on the central fibre, $p_{1}(0)$ and $p_{9}(0) \in \mathbb{F} \cap \mathbb{P}$.

Consider $\widetilde{\mathcal{X}}^{\prime \prime}$ the blow-up of $\mathcal{X}^{\prime \prime}$ along the horizontal curves $\left\{p_{i}(s)\right\}_{s \in \Delta^{\prime}}$, with $\mathcal{E}_{i}$ exceptional divisors, $i=1, \ldots, 9$. The components of the fibres are described by the same Picard 
groups as the components of $\widetilde{X}_{0}^{\prime}$ (see Sect. 3.1.1). We use the symbols $\mathbb{F}_{0}$ and $\mathbb{P}_{0}$ to denote the pullbacks of the components of the central fibre over $\Delta^{\prime}, X_{0}^{\prime \prime}$, and the symbol $Y_{0}$ for their intersection. Notice that $Y_{0} \cong \mathrm{Bl}_{p_{1}, p_{9}}\left(\mathbb{P}^{2}\right)$ is a plane blown-up at two points.

The combination of the two above subsequent degenerations produces a degeneration of $\mathrm{Bl}_{p_{1}, \ldots, p_{9}}\left(\mathbb{P}^{3}\right)$ to the union of blown-up spaces $\widetilde{X}_{0}^{\prime \prime}=\mathbb{F}_{0} \cup \mathbb{P}_{0}$ intersecting along a blown-up plane $Y_{0}$.

\subsubsection{Intersection table on the central fibre}

Notice that, as a divisor on $\mathbb{F}_{0}\left(\right.$ or on $\left.\mathbb{P}_{0}\right)$, the surface $Y_{0}$ is represented by the class $E_{0}-E_{9}$ (resp. $H^{\mathbb{P}}-E_{1}$ ).

One can compute the restrictions of any divisor on $\mathbb{F}_{0}$ or on $\mathbb{P}_{0}$ to $Y_{0}$, by means of the following intersection table for the generators of the Picard groups:

- $\left.H^{\mathbb{P}}\right|_{Y_{0}}=: h$,

- $\left.E_{1}\right|_{Y_{0}}=: e_{1}$,

- $\left.E_{2}\right|_{Y_{0}}=0$,

and

- $\left.H^{\mathbb{F}}\right|_{Y_{0}}=0$,

- $\left.E_{0}\right|_{Y_{0}}=-h$,

- $\left.E_{i}\right|_{Y_{0}}=0, i=3, \ldots, 8$,

- $\left.E_{9}\right|_{Y_{0}}=: e_{9}$.

In this notation, we have $\operatorname{Pic}\left(Y_{0}\right)=\left\langle h, e_{1}, e_{9}\right\rangle$.

\subsection{The limit linear system}

Let $\mathcal{L} \subset\left|\mathcal{O}_{\mathbb{P}^{3}}(d)\right|$ be a linear system of degree- $d$ surfaces in $\mathbb{P}^{3}$ with nine assigned multiple points in general position. Let $D$ be the corresponding divisor in the blown-up space $\mathrm{Bl}_{p_{1}, \ldots, p_{9}}\left(\mathbb{P}^{3}\right)$.

In the notation of Sect. 3.1.1, consider on $\mathcal{X}^{\prime}$ the twisted line bundle $\mathcal{O}_{\mathcal{X}^{\prime}}(d) \otimes \mathcal{O}_{\mathcal{X}^{\prime}}(-\delta \mathbb{P})$. It restricts to $\mathcal{O}_{\mathbb{P}^{3}}(d)$ on $X_{t}^{\prime}$ and, for $t=0$, to $\mathcal{O}_{\mathbb{F}}\left(d H_{\mathbb{F}}-\delta E_{0}\right)$ on $\mathbb{F}$ and to $\mathcal{O}_{\mathbb{P}}\left(\delta H^{\mathbb{P}}\right)$ on $\mathbb{P}$. By following the first degeneration, we can consider the linear system $\mathcal{L}_{t}=\mathcal{L} \subset\left|\mathcal{O}_{\mathbb{P}^{3}}(d)\right|$ on $X_{t}^{\prime} \cong \mathbb{P}^{3}$ and its limit $\mathcal{L}_{0}^{\prime}$ on $X_{0}^{\prime}$. We denote by $D_{0}^{\prime}$ the corresponding divisor class in the blown-up central fibre $\tilde{X}_{0}^{\prime}$.

By following the second degeneration and blowing-up the nine points on each fibre (see Sect. 3.1.2), we obtain the limit divisor $D_{0}^{\prime \prime}$ in the blown-up central fibre $\tilde{X}_{0}^{\prime \prime}$ that is given by divisors $D^{\mathbb{F}_{0}}$ and $D^{\mathbb{P}_{0}}$ on the two components. We consider the restriction maps to $Y_{0}, D^{\mathbb{F}_{0}} \rightarrow$ $R^{\mathbb{F}_{0}}:=\left.D^{\mathbb{F}_{0}}\right|_{Y_{0}}$ and $D^{\mathbb{P}_{0}} \rightarrow R^{\mathbb{P}_{0}}:=\left.D^{\mathbb{P}_{0}}\right|_{Y_{0}}$ and denote by $\hat{D}^{\mathbb{F}_{0}}$ and $\hat{D}^{\mathbb{P}_{0}}$ the kernels, respectively. Let $R_{0}:=R^{\mathbb{F}_{0}} \cap R^{\mathbb{P}_{0}}$ denote the intersection of the restricted divisors.

Lemma 3.4 In the notation of above, for $i \geq 0$, we have

$$
h^{i}\left(\widetilde{X}_{0}^{\prime \prime}, D_{0}^{\prime \prime}\right)=h^{i}\left(\mathbb{P}_{0}, \hat{D}^{\mathbb{P}_{0}}\right)+h^{i}\left(\mathbb{F}_{0}, \hat{D}^{\mathbb{F}_{0}}\right)+h^{i}\left(Y_{0}, R_{0}\right) .
$$

Proof Notice first of all that the assertion holds if we replace $h^{i}$ by $\chi$, the Euler characteristic. The equality holds for $i=0$ by construction. Indeed, the divisor $D_{0}^{\prime \prime}$ on $\widetilde{X}_{0}^{\prime \prime}$, or its associated line bundle, is obtained as fibred product of $D^{\mathbb{F}_{0}}$ and $D^{\mathbb{P}_{0}}$ over $R_{0}$, see Sect. 2.1. Finally, since all cohomology groups with $i \geq 2$ vanish, the assertion holds for $i=1$. 
Lemma 3.5 In the notation of above, we have

$$
h^{i}\left(B l_{p_{1}, \ldots, p_{s}}\left(\mathbb{P}^{n}\right), D\right) \leq h^{i}\left(\widetilde{X}_{0}^{\prime}, D_{0}^{\prime}\right) \leq h^{i}\left(\widetilde{X}_{0}^{\prime \prime}, D_{0}^{\prime \prime}\right), \quad i=0,1 .
$$

Proof The inequalities hold for $i=0$ by the property of upper semi-continuity of the two degenerations. As $\chi\left(\mathrm{Bl}_{p_{1}, \ldots, p_{9}}\left(\mathbb{P}^{3}\right), D\right)=\chi\left(\widetilde{X}_{0}^{\prime}, D_{0}^{\prime}\right)=\chi\left(\widetilde{X}_{0}^{\prime \prime}, D_{0}^{\prime \prime}\right)$ and all higher cohomology groups vanish, the inequalities hold for $i=1$ as well.

Remark 3.6 The above construction as well as Lemma 3.4 and Lemma 3.5 is potentially applicable in a more general context for linear systems in any $\mathbb{P}^{n}$ and with arbitrary number of points and multiplicities by choosing different specializations and twists, as it was done, for instance, by the last author in [30]. Nevertheless, it is not easy to find a good degeneration in general.

\subsection{Proof of Theorem 3.1}

In order to prove the theorem, we need the following result.

Proposition 3.7 The following linear systems are non-special with dimension equal to the virtual dimension: $\mathcal{L}_{3,2 m}\left(m+1, m^{6}, m-1\right), \mathcal{L}_{3,2 m-1}\left(m^{4},(m-1)^{4}\right)$.

This can be easily deduced from [15] where the authors deal with Cremona reduced linear systems with eight base multiple points. However, we include the proof here for the sake of completeness.

Proof One can easily check that $\operatorname{vdim}\left(\mathcal{L}_{3,2 m}\left(m+1, m^{6}, m-1\right)\right)=0$. The statement is obviously true for $m=1$. By performing two subsequent Cremona transformations of $\mathbb{P}^{3}$ (see Sect. 2.3), we reduce from $m$ to $m-1$. Hence, we conclude by induction on $m$.

Similarly, one proves that $\operatorname{dim}\left(\mathcal{L}_{3,2 m-1}\left(m^{4},(m-1)^{4}\right)\right)=\operatorname{vdim}\left(\mathcal{L}_{3,2 m-1}\left(m^{4},(m-\right.\right.$ $\left.\left.1)^{4}\right)\right)=0$ by induction on $m$.

Proof of Theorem 3.1 Let $Q=\mathcal{L}_{3,2}\left(1^{9}\right)$ be the quadric surface through the nine base points. The obvious inclusion of linear systems $\mathcal{L}-a Q \subseteq \mathcal{L}$ implies the inequality $\operatorname{dim}(\mathcal{L}-a Q) \leq$ $\operatorname{dim}(\mathcal{L})$. But $\mathcal{L}-a Q=\mathcal{L}_{3,2(m-a)}\left((m-a)^{8}\right)$ and, by Proposition 3.7, $\operatorname{dim}\left(\mathcal{L}_{3,2(m-a)}((m-\right.$ $\left.\left.a)^{8}\right)\right)=\operatorname{vdim}\left(\mathcal{L}_{3,2(m-a)}\left((m-a)^{8}\right)\right)=m-a+1 \geq 1$. Hence, $m-a+1 \leq \operatorname{dim}(\mathcal{L}(m, a))$.

We prove the inverse inequality by degeneration. Let $D$ denote the divisor in $\mathrm{Bl}_{p_{1}, \ldots, p_{9}}\left(\mathbb{P}^{3}\right)$ corresponding to $\mathcal{L}(m, a)$ :

$$
D=2 m H-m \sum_{i=1}^{8} E_{i}-a E_{9}
$$

In the notation of Sect. 3.2, now with $d=2 m$, choose $\delta=m$. In the space $\tilde{\mathcal{X}}^{\prime \prime}$ of the second degeneration, we have the following divisors on the components of the central fibre $\widetilde{X}_{0}^{\prime \prime}$ :

$$
\begin{aligned}
& D^{\mathbb{F}_{0}}=2 m H^{\mathbb{F}}-m E_{0}-m \sum_{i=1}^{7} E_{i}, \\
& D^{\mathbb{P}_{0}}=m H^{\mathbb{P}}-m E_{8}-a E_{9} .
\end{aligned}
$$

We consider the restriction maps to $Y_{0}$, and we obtain the following kernel divisors

$$
\hat{D}^{\mathbb{F}_{0}}=2 m H^{\mathbb{F}}-(m+1) E_{0}-(m-1) E_{1}-m \sum_{i=2}^{7} E_{i},
$$




$$
\hat{D}^{\mathbb{P}_{0}}=(m-1) H^{\mathbb{P}}-m E_{8}-(a-1) E_{9} .
$$

Firstly, on the component $\mathbb{F}_{0}$ of the central fibre, we have the following. By Proposition 3.7, we obtain that both $D^{\mathbb{F}_{0}}$ and $\hat{D}^{\mathbb{F}_{0}}$ are non-special, so that the first cohomology groups vanish; moreover, the second is non-effective, namely $h^{0}=0$.

Secondly, on the exceptional component of $\widetilde{X}_{0}^{\prime \prime}$, we have the following. The divisor $D^{\mathbb{P}_{0}}$ is (only) linearly obstructed and has $h^{1}\left(D^{\mathbb{P}_{0}}\right)=\left(\begin{array}{c}a+1 \\ 3\end{array}\right)$ caused by a line of multiplicity $a$, see [5]. Moreover, the kernel $\hat{D}^{\mathbb{P}_{0}}$ is non-effective and has $h^{1}\left(\hat{D}^{\mathbb{P}_{0}}\right)=\left(\begin{array}{c}a+1 \\ 3\end{array}\right)$, see [18].

The above implies that both $D^{\mathbb{F}_{0}}$ and $D^{\mathbb{P}_{0}}$ cut the complete linear series on the intersection $Y_{0}$ of the components. Using Sects. 3.1.3 and 3.2 and the notation there introduced, we have

$$
R^{\mathbb{P}_{0}}=m h-a e_{9}, \quad R^{\mathbb{F}_{0}}=m h-m e_{1} .
$$

Since $R^{\mathbb{F}_{0}}$ and $R^{\mathbb{P}_{0}}$ meet transversally on $Y_{0}$, their intersection is given by

$$
R_{0}=m h-m e_{1}-a e_{9} .
$$

One computes $h^{0}\left(R_{0}\right)=m-a+1$ and $h^{1}\left(R_{0}\right)=\left(\begin{array}{l}a \\ 2\end{array}\right)$, the speciality being given by a line of multiplicity $a$, see [5]. Finally, by Lemma 3.4, we obtain $h^{0}\left(D_{0}^{\prime \prime}\right)=m-a+1$ and $h^{0}\left(D_{0}^{\prime \prime}\right)=\left(\begin{array}{c}a+1 \\ 3\end{array}\right)+\left(\begin{array}{l}a \\ 2\end{array}\right)$. Now we conclude the proof of the first part of the theorem by upper semi-continuity, see Lemma 3.5 .

To prove the last sentence of the theorem, we simply notice that the linear system $\mathcal{L}(m, a)$ splits as follows:

$$
\mathcal{L}(m, a)=a Q+\mathcal{L}_{3,2(m-a)}\left((m-a)^{8}\right) .
$$

The second addend in the right-hand side is the moving part of $\mathcal{L}$ and is non-special by Proposition 3.7. This concludes the proof.

Remark 3.8 In the proof of Theorem 3.1, we argued that the speciality of $\mathcal{L}(m, a)$ is given by $a Q$ and equals the speciality of the limit $D_{0}^{\prime \prime}$ that, using Lemma 3.4, is given by a line of multiplicity $a$ in the base locus of $\hat{D}^{\mathbb{P}_{0}}$ and a line of multiplicity $a$ in the base locus of $R_{0}$.

A geometric interpretation is the following. Let us denote by $\mathcal{L}^{\mathbb{P}_{0}, \mathbf{m}}$ the matching linear system defined by the matching conditions imposed by $R^{\mathbb{F}_{0}}$ to $R^{\mathbb{P}_{0}}$, so that we have the following exact sequence of sheaves

$$
0 \rightarrow\left|\hat{D}^{\mathbb{P}_{0}}\right| \rightarrow \mathcal{L}^{\mathbb{P}_{0}, \mathbf{m}} \rightarrow\left|R_{0}\right| \rightarrow 0 .
$$

The emptiness of $\left|\hat{D}^{\mathbb{P}_{0}}\right|$ implies that the limit linear system $\left|D_{0}^{\prime \prime}\right|$ is the matching linear system; it is of the form $\mathcal{L}_{3, m}(m, m, a)$ where the second and third points, $p_{8}, p_{9}$, are general in $\mathbb{P}_{0}$, while the first point, $p_{1}$, is on the intersection and is the one giving the matching.

In particular, if we follow the quadric $Q=\mathcal{L}(1,1)$ in the degeneration process, by simply setting $m=a=1$ in the above, we see that its limit is given by a matching linear system on $\mathbb{P}_{0}$ of the form $\mathcal{L}_{3,1}(1,1,1)$, based at the points $p_{8}, p_{9}$ and $p_{1}$ of the central fibre as above. This linear system has only one element that is the plane spanned by the three points. This plane is the special effect variety for the limit of $\mathcal{L}(m, a)$; it is contained with multiplicity $a$ in the base locus and it contributes by $\left(\begin{array}{c}a+1 \\ 3\end{array}\right)+\left(\begin{array}{l}a \\ 2\end{array}\right)$ to the speciality.

A weak base locus lemma for the quadric $Q$ in the case of $\mathcal{L}(m, a)$ is just an easy application of Lemma 2.1. Indeed to prove that $Q$ is contained in the base locus of $\mathcal{L}(m, a)$ with multiplicity at least $a$, it is enough to show that for every $m$ and $a$, the restriction $\left.\mathcal{L}(m, a)\right|_{Q}$ is empty and this is equivalent to prove that $\mathcal{L}_{2,3 m}\left(m^{9}, a\right)$ is empty, which is a well-known fact. 
In the next section, we will see that in general to obtain such a result is extremely difficult, mostly because of the very little knowledge of linear systems on $\mathbb{P}^{1} \times \mathbb{P}^{1}$.

\section{Homogeneous linear systems $\mathcal{L}_{3,2 m+1}\left(m^{9}\right)$}

In this section, we consider linear systems with nine points of multiplicity $m$ and degree $2 m+1$. For this class of linear system, it is more difficult to understand the relation between the speciality and the presence of the quadric as special effect variety. Even to compute the multiplicity of containment of the quadric in the base locus is not an obvious task. The main result of this section is in fact a vanishing result for linear systems in $\mathbb{P}^{2}$ which allows to deduce a base locus lemma for the quadric.

Given a linear system $\mathcal{L}=\mathcal{L}_{3, d}\left(m_{1}, \ldots, m_{s}\right)$ with $s \geq 9$, let $Q$ be the unique quadric surface through the first nine points. Consider the restriction exact sequence

$$
0 \rightarrow \mathcal{L}-Q \rightarrow \mathcal{L} \rightarrow \mathcal{L}_{\mid Q} \rightarrow 0 .
$$

The linear system $\mathcal{L}_{\mid Q}$ is contained in the linear system of the curves of bidegree $(d, d)$ in $Q \cong \mathbb{P}^{1} \times \mathbb{P}^{1} \subset \mathbb{P}^{3}$ with nine multiple points and denoted by $\mathcal{L}_{\mathbb{P}^{1} \times \mathbb{P}^{1},(d, d)}\left(m_{1}, \ldots, m_{9}\right)$ as in Sect. 2.2.

By Lemma 2.1, we know that the system $\mathcal{L}_{\mathbb{P}^{1} \times \mathbb{P}^{1}(d, d)}\left(m_{1}, \ldots, m_{9}\right)$ has the same dimension as the system $\mathcal{L}_{2,2 d-m_{1}}\left(d-m_{1}, d-m_{1}, m_{2}, \ldots, m_{s}\right)$ in $\mathbb{P}^{2}$, and in particular, the first system is empty if and only if the second one is.

The main part of this chapter is devoted to prove, via degeneration techniques, emptiness results for linear systems in $\mathbb{P}^{2}$ with ten multiple points. As a straightforward consequence of Theorem 4.2 below, we obtain the following (weak) base locus lemma for the quadric. Let $\alpha$ be any positive integer.

Theorem 4.1 (Quadric base locus lemma) Let $\mathcal{L}=\mathcal{L}_{3,2 m+\alpha}\left(m^{9}, m_{10} \ldots, m_{s}\right)$ be a nonempty linear system. If $m>9 \alpha$, then the quadric $Q$ through the first nine points is contained in the base locus of $\mathcal{L}$.

We remark that a major difference between the quadric through nine points in $\mathbb{P}^{3}$ and the linear cycles in $\mathbb{P}^{n}$ is in the geometry of their normal bundles. For the last ones, the normal bundles are toric bundles so we understand their cohomology groups [5,18], while for the first one, the cohomological information is highly non-trivial.

\subsection{Emptiness of linear systems with ten points in $\mathbb{P}^{2}$}

The goal of this section is to find a good bound for $m$ to have emptiness of certain linear systems in $\mathbb{P}^{2}$. More precisely, we will prove the following result, which implies Theorem 4.1.

Theorem 4.2 The linear system $\mathcal{L}=\mathcal{L}_{2,3 m+2 \alpha}\left((m+\alpha)^{2}, m^{8}\right)$ is empty for any $m>9 \alpha$.

We will prove this result via degeneration techniques similar to the ones introduced in Sect. 3.1. More precisely, we will simultaneously degenerate the blown-up projective plane at ten points in general position and the line bundle $\mathcal{L}$. Even though this technique was applied before in $[10,13]$ for homogeneous linear systems with ten points, we will present here in detail our approach. 


\subsubsection{The first degeneration}

By blowing-up a point in the central fibre of a trivial family of projective planes over a disc, $\Delta$, one obtains a new family, call it $\mathcal{X} \rightarrow \Delta$. The fibre over zero, $X_{0}$, decomposes as the union of two surfaces, a projective plane denoted by $\mathbb{P}$ and the Hirzebruch surface $\mathbb{F}_{1}$, call it $\mathbb{F}$. In this notation, $\mathbb{P}$ represents the exceptional divisor of the blown-up point, while $\mathbb{F}$ is the proper transform of the central fibre of the original family. We will denote by $E$ the curve of intersection between $\mathbb{P}$ and $\mathbb{F}$.

Consider now ten points on the general fibre of the trivial family of planes, such that four of them collide in the zero fibre. Correspondingly, on the central fibre of $\mathcal{X}$, we place six points on $\mathbb{F}$ and four points on $\mathbb{P}$ and we consider them as ten limit points of general points on $X_{t}$. Blowing-up these ten sections of $\mathcal{X}$ creates a new family $\mathcal{X}^{\prime} \rightarrow \Delta$. The fibre over zero consists of two surfaces, $\mathbb{P}_{0}$ and $\mathbb{F}_{0}$, that intersect along a double curve, $E$. The component $\mathbb{P}_{0}$ represents a blown-up plane at four general points, $\mathbb{F}_{0}$ represents the blown-up ruled surface $\mathbb{F}_{1}$, at six general points, while the double curve $E$ is the negative section on the component $\mathbb{F}_{0}$ and also represents the class of a line on $\mathbb{P}_{0}$. The general fibre $X_{t}^{\prime}$ is the blown-up projective plane at ten general points.

Remark 4.3 We point out that colliding four points in the zero fibre works well for the analysis of linear systems with ten points. In general, by colliding $s^{\prime}$ points from a collection of $s$ general points, one produces a degeneration of the blown-up projective plane at $s$ general points, i.e. the general fibre $X_{t}^{\prime}$, to the union of two surfaces (for any choice of $s^{\prime}$ and $s$ ). The components of the central fibre are as follows: $\mathbb{P}_{0}$ that is a blown-up plane at $s^{\prime}$ general points and $\mathbb{F}_{0}$ that is the blown-up ruled surface $\mathbb{F}_{1}$ at $s-s^{\prime}$ general points, the two surfaces meeting along a double curve.

\subsubsection{The second degeneration}

This degeneration was first introduced in [13], and we provide the construction of the degeneration together with the limit bundles computation for the sake of completeness. The interested reader should also consult [10]. We denote by $C$ the unique (-1)-curve on $\mathbb{F}$ passing through six points that meet the double curve $E$ in two points $p_{1}$ and $p_{2}$, at the form $\mathcal{L}_{3}\left(2,1^{6}\right)$. We consider the family obtained in Sect. 4.1.1, $\mathcal{X}^{\prime} \rightarrow \Delta$, and we blow-up twice the cubic $C$ on $\mathbb{F}$ and then contract the first exceptional divisor created. In this way, we will obtain a new family $\mathcal{X}^{\prime \prime} \rightarrow \Delta$ whose general fibre is still a plane blown-up at ten points and whose special fibre over the origin becomes the union of four surfaces. We abuse notations and denote by $\mathbb{F}$ and $\mathbb{P}$ the surfaces of the central fibre in the second degeneration and by $\mathbb{S}$ and $\mathbb{T}$ the exceptional divisors created by the double blow-up of $C$.

The first blow-up of $C$ in the threefold $\mathcal{X}^{\prime}$ creates as exceptional divisor a Hirzebruch surface $\mathbb{F}_{1}$ that we will denote by $\mathbb{T}$. The rational curve $C$ represents the intersection between $\mathbb{T}$ and $\mathbb{F}$. In particular, $C$ represents the $(-1)$-curve of $\mathbb{T}$, while $G_{i}$ represent the fibre class on $\mathbb{T}$, see Fig. 2.

The second blow-up of $C$ in $\mathcal{X}$, creates the exceptional divisor $\mathbb{S}$ that is isomorphic to $\mathbb{P}^{1} \times \mathbb{P}^{1}$ and blows-up the surface $\mathbb{P}$ twice. Denote by $F_{1}$ and $F_{2}$, the exceptional divisors introduced on $\mathbb{P}$. Notice that the proper transforms of $G_{1}$ and $G_{2}$ become (-2)-curves. We abuse the notation and denote by $G_{i}$ to be these proper transforms with self-intersection $(-2)$.

Since the normal bundle of $\mathbb{S}$ has bidegree $(-1,-1)$, one can contract the ruling direction of $\mathbb{S}$. Blowing-down $\mathbb{S}$ will affect the surfaces of the central fibre as follows. On the surface $\mathbb{F}$, the cubic $C$ will get contracted, $\mathbb{T}$ will become a projective plane, while on $\mathbb{P}$ the $(-1)$-curves, 


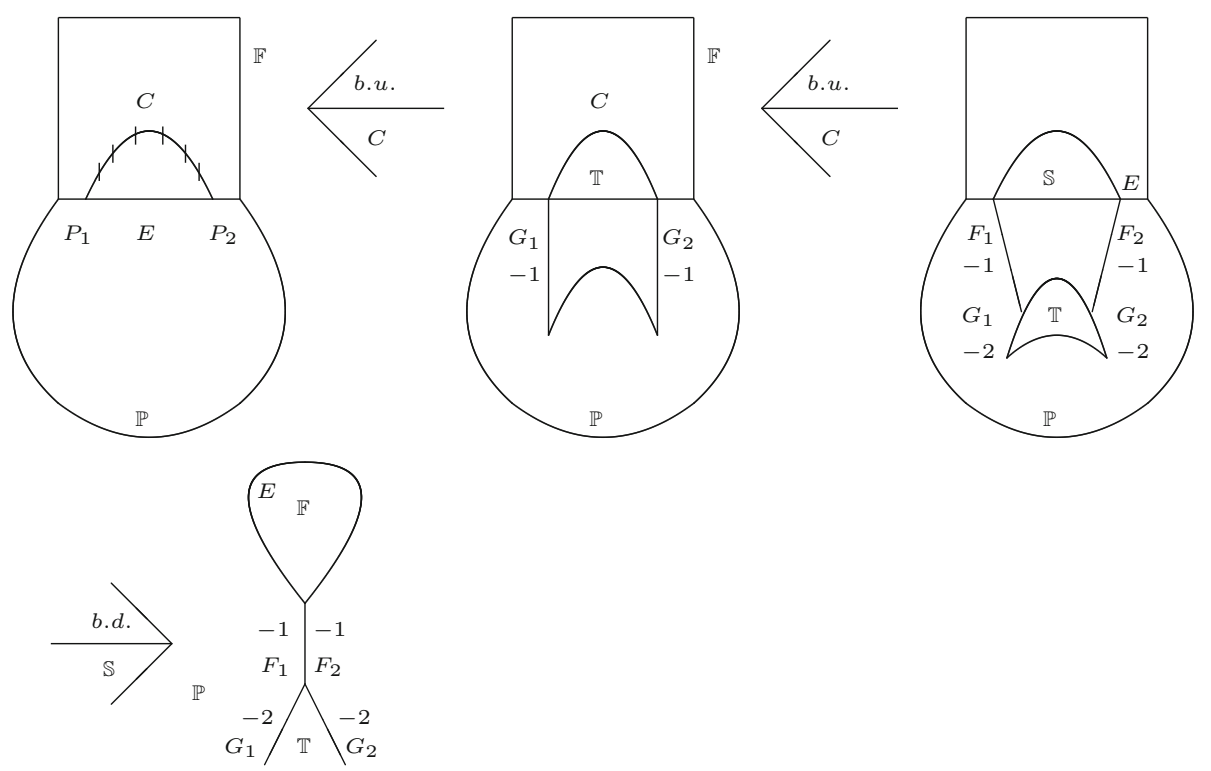

Fig. 2 The 2-throw operation of the double curve $C$

$F_{1}$ and $F_{2}$, will get identified. In $[10,13]$, this operation is called a 2-throw of $C$ on $\mathbb{P}$, see again Fig. 2.

\subsubsection{Degenerating the line bundles}

We will now describe limits of line bundles on $\mathbb{P}^{2}$ via the double degeneration. The limit bundles are bundles in the central fibre, $X_{0}^{\prime \prime}$, of the family $\mathcal{X}^{\prime \prime} \rightarrow \Delta$ that agree on the intersection of the double curves.

Remark 4.4 The double blow-up of $C$ in $\mathcal{X}$ affected the surface $\mathbb{P}$ by creating two pairs of points that are infinitely near. We will use the notation of [13] $\left[m_{1}, m_{2}\right]$, to indicate a fat point with multiplicity $m_{1}$ and an infinitely near fat point with multiplicity $m_{2}$. More precisely, we adopt the notation $\left[m_{1}, m_{2}\right]$ to denote $m_{2} F_{1}+m_{1}\left(F_{1}+G_{1}\right)$.

Remark 4.5 In this section, as in Sect. 3.2, we will describe limits of divisors, and not limits of linear systems. This difference is emphasized in Remark 4.4. In particular, Proposition 4.6 should be understood as describing all possible limit divisors $D$ in the linear system $\mathcal{L}_{2, d}((m+$ $\alpha)^{2}, m^{8}$ ) on the central fibre $X_{0}^{\prime \prime}$. However, in order to simplify the language and also to be consistent with notation previously used in $[10,13]$, in this section we abuse notations and we use the linear system terminology. We must also emphasize now that this degeneration is different than the one we exploited in Sect. 3.2. More precisely, only the first degeneration of the blown-up projective space $\mathbb{P}^{3}$ described in Sects. 3.2 and 4.1.1 coincide. In Sect. 3.2, this degeneration was denoted by $\widetilde{\mathcal{X}}^{\prime}$, while in Sect. 4.1.1, it was denoted by $\mathcal{X}^{\prime}$. However, in Sect. 3.2 the second degeneration was obtained by specializing points on the intersection of the two components, while in Sect. 4.1.2, the second degeneration is obtained from flopping a negative curve. In order to highlight this major difference, we choose different notations. More precisely, even if both represent degenerations of blown-up projective projective spaces 
$\left(\mathbb{P}^{3}\right.$ in Sect. 3.2 and $\mathbb{P}^{2}$ here), we will denote them by $\widetilde{\mathcal{X}}^{\prime \prime}$ and $\mathcal{X}^{\prime \prime}$ in Sects. 3.2 and 4.1.2, respectively.

We will now determine all possible limit bundles of the linear system

$$
\mathcal{L}_{2, d}\left((m+\alpha)^{2}, m^{8}\right)
$$

on the general fibre.

- The line bundle on $\mathbb{P}$ must be of the form $\mathcal{L}_{\delta}\left(m^{4},[a, b],[a, b]\right)$, where $\delta, a, b$ represent the twisting parameters. This line bundle meets the four times blown-up line $\delta-2 a-2 b$ times, see Remark 4.7 below. The system on $\mathbb{F}$ is of the form $\mathcal{L}_{\mathbb{F}}=\mathcal{L}_{2, t}\left(\delta-2 a-2 b, y^{4}, y^{\prime 2}\right)$, for some $t, y, y^{\prime}$. The assumption that $\mathcal{L}_{\mathbb{F}}$ does not meet the cubic $C$ implies that the degree of $\mathcal{L}_{\mathbb{F}}$ has to be even, write $t=2 e$. Indeed $0=\mathcal{L}_{\mathbb{F}} \cdot C=3 t-2 \delta+4 a+4 b-4 y-2 y^{\prime}$. Moreover, because $y=m-a-b$ and $y^{\prime}=m-a-b+\alpha$, then one can check that

$$
\delta=3 e-3 m+5 a+5 b-\alpha .
$$

- Consider the intersection of $\mathbb{S}$ and $\mathbb{F}$ that is a fibre on $\mathbb{S}$ and the cubic on $\mathbb{F}$. Note that $\mathcal{L}_{\mathbb{S}}$ is a horizontal bundle so it must have bidegree $(b, 0)$. Moreover, $\mathcal{L}_{\mathbb{T}}$ meets a fibre $a-b$ times and does not meet the negative section $B$. Hence,

$$
\mathcal{L}_{\mathbb{T}}=\mathcal{L}_{2, a-b} .
$$

- The last parameter to be determined is $e$. We compute it by observing that the limit bundle should have degree $d$, that is the degree on the bundle on the general fibre. By pulling-back a line in the plane, we get a line on $\mathbb{P}$, a fibre on $\mathbb{F}$, a fibre on $\mathbb{T}$ and a fibre on $\mathbb{S}$. Therefore, the intersection number with all the bundles from above will have to add up to $d$. We obtain

$$
e=\frac{d-3(a+b)}{2}
$$

Solving this system of linear equations, we obtain

$$
\mathcal{L}_{\mathbb{F}}=\mathcal{L}_{2, d-3 a-3 b}\left(\frac{3 d}{2}-3 m-\frac{3(a+b)}{2}-\alpha,(m-a-b)^{4},(m-a-b+\alpha)^{2}\right) .
$$

The surface $\mathbb{S}$ will be contracted in the ruling direction. This last blow-down will affect the surface $\mathbb{F}$ by contracting the cubic $C$ to a point by performing a series of Cremona transformations to $\mathcal{L}_{\mathbb{F}}$, see Sect. 2.3. After contracting the surface $\mathbb{S}$, the bundle on $\mathbb{F}$ becomes

$$
\mathcal{L}_{\mathbb{F}}=\mathcal{L}_{2,3 m-\frac{d}{2}-\frac{3(a+b)}{2}+\alpha}\left(0,\left(2 m-\frac{d}{2}-\frac{a+b}{2}+\alpha\right)^{4},\left(2 m-\frac{d}{2}-\frac{a+b}{2}\right)^{2}\right) .
$$

The zero multiplicity of $\mathcal{L}_{\mathbb{F}}$ represents the image of the cubic after the Cremona transformations. Since we are contracting the surface $\mathbb{S}$, we will simply ignore this multiplicity. We recall that contracting the cubic on $\mathbb{F}$ will also affect the surface on $\mathbb{P}$ by identifying the two last (-1)-curves created on $\mathbb{P}$, namely $F_{1}$ and $F_{2}$.

For the future analysis, we will work with the normalization of $\mathbb{P}$, so we will consider $F_{1}$ and $F_{2}$ disjoint as before. We obtain the following result.

Proposition 4.6 All limits of the bundle $\mathcal{L}_{d}\left((m+\alpha)^{2}, m^{8}\right)$ are of the following form, for some choice of the parameters $a$ and $b$ : 
- $\mathcal{L}_{\mathbb{P}}=\mathcal{L}_{2, \frac{3 d}{2}-3 m+\frac{a+b}{2}-\alpha}\left(m^{4},[a, b],[a, b]\right)$,

- $\mathcal{L}_{\mathbb{F}}=\mathcal{L}_{2,3 m-\frac{d}{2}-\frac{3(a+b)}{2}+\alpha}\left(\left(2 m-\frac{d}{2}-\frac{a+b}{2}+\alpha\right)^{4},\left(2 m-\frac{d}{2}-\frac{a+b}{2}\right)^{2}\right)$,

- $\mathcal{L}_{\mathbb{T}}=\mathcal{L}_{2, a-b}$.

Remark 4.7 We describe here how our degeneration method works. The choice of the number of points that one collides in the central fibre in the first degeneration (in this case is four, see Sect. 4.1.1) determines at each step of the degeneration the (-1)-curves that one must flop in order to obtain better ratios for proving emptiness or non-speciality results. In the analysis of linear systems with ten points, both approaches of proving emptiness or non-speciality lead to the same degeneration, see [10] and [13].

Namely, in any degeneration one computes a fixed limit ratio $\frac{d}{m}$ such that for any choice of the twisting parameters, all line bundles on the central fibre are non-empty. But this ratio satisfies the Nagata bound, so one of the linear systems is special. According to Segre-Harbourne-Gimigliano-Hirschowitz Conjecture, this effective linear system is special because of the existence of a negative curve. In the first degeneration, this curve is precisely the special cubic passing through seven points, denoted by $C$, that distinguishes by splitting off the line bundle on $\mathbb{P}$. To improve the limit ratio, $\frac{d}{m}$, one needs to flop the curve creating speciality.

In general, if the curve creating speciality of one of the linear systems of the central fibre intersects the double curve $E$ once, then one performs a one-throw as explained in [13]. In other words, by blowing-up the curve creating speciality, the exceptional divisor introduced is a ruled surface isomorphic to $\mathbb{P}^{1} \times \mathbb{P}^{1}$, so one can contract the other ruling.

However in our case, the special curve is the cubic $C$ intersecting the double curve $E$ twice. It follows by the general intersection theory that the exceptional divisor created after the first blow-up, denoted by $\mathbb{T}$, is a Hirzebruch surface $\mathbb{F}_{1}$ that can not be contracted. So a second blow-up is necessary creating two infinitely near points; the new exceptional divisor, denoted by $\mathbb{S}$, is the ruled surface $\mathbb{P}^{1} \times \mathbb{P}^{1}$. This can be seen by intersection theory of surfaces in $\mathbb{P}^{3}$. This affects the double curve of intersection, transforming $E$ to the strict transform of a line blownup four times. Finally, the fibre direction of $\mathbb{S}$ can be blown-down. This blow-down simplifies the geometry of $\mathbb{F}$, but it increases the difficulty of the study of the linear system on $\mathbb{P}$.

We would like to point our that each degeneration is uniquely determined by the number of points we decide to collide in the first step, the degree and multiplicities of the linear system. In this case, the same degeneration as in [10] and [13] can be applied, but for the sake of simplicity, the computations that lead to this degeneration were omitted.

We study now the effectivity of $\mathcal{L}_{\mathbb{P}}$ and $\mathcal{L}_{\mathbb{F}}$ for $d=3 m+2 \alpha$ and $m \geq 8 \alpha$. Notice that by substituting $d=3 m+2 \alpha$, we obtain the following bundles on $\mathbb{P}$ and $\mathbb{F}$ :

$$
\begin{aligned}
& \mathcal{L}_{\mathbb{P}}=\mathcal{L}_{2, \frac{3 m}{2}+\frac{a+b}{2}+2 \alpha}\left(m^{4},[a, b],[a, b]\right), \\
& \mathcal{L}_{\mathbb{F}}=\mathcal{L}_{2, \frac{3 m}{2}-\frac{3(a+b)}{2}}\left(\left(\frac{m}{2}-\frac{a+b}{2}\right)^{4},\left(\frac{m}{2}-\frac{a+b}{2}-\alpha\right)^{2}\right) .
\end{aligned}
$$

Remark 4.8 The following two statements are obvious.

- The linear system on $\mathbb{T}, \mathcal{L}_{\mathbb{T}}=\mathcal{L}_{2, a-b}$, is nonempty if and only if $a \geq b$.

- The linear system on $\mathbb{F}, \mathcal{L}_{\mathbb{F}}$ is non-empty if any only if $a+b \leq m$.

We will now analyse the linear system on $\mathbb{P}$. We denote by $Q_{i}$ the four quartics $\mathcal{L}_{4}\left(2^{3}, 1,[1,1]^{2}\right)$ on $\mathbb{P}$ and we see that these $(-1)$-curves split off the system if $m \geq 8 \alpha$. Indeed, 


$$
\begin{aligned}
\mathcal{L}_{\mathbb{P}} Q_{i} & =\mathcal{L}_{2, \frac{3 m}{2}+\frac{a+b}{2}+2 \alpha}\left(m^{4},[a, b],[a, b]\right) \mathcal{L}_{2,4}\left(2^{3}, 1,[1,1]^{2}\right) \\
& =4\left(\frac{3 m}{2}+\frac{a+b}{2}+2 \alpha\right)-3 \cdot 2 \cdot m-m-2 a-2 b=8 \alpha-m .
\end{aligned}
$$

We further apply a series of four Cremona transformations to the linear system $\mathcal{L}_{\mathbb{P}}$ (which contains eight base points $\left.p_{1}, \ldots, p_{8}\right)$ based, respectively, at the points $\left\{p_{1}, p_{2}, p_{3}\right\},\left\{p_{4}, p_{5}, p_{8}\right\}$, $\left\{p_{4}, p_{6}, p_{7}\right\}$ and $\left\{p_{1}, p_{2}, p_{3}\right\}$. Note that this series of Cremona transformations contracts the four quartics to a point at the same time.

$$
\begin{aligned}
& \operatorname{Cr}\left(\mathcal{L}_{\mathbb{P}}\right)=\mathcal{L}_{2, \frac{a+b}{2}-\frac{5 m}{2}+18 \alpha}\left((8 \alpha-m)^{4},\right. \\
& \quad[a-m+4 \alpha, b-m+4 \alpha],[a-m+4 \alpha, b-m+4 \alpha]) .
\end{aligned}
$$

For $m \geq 8 \alpha$, the exceptional divisors corresponding to the first four points are (-1)-curves that split off the system. These exceptional divisors represent the four quadrics; we will remove them and forget the zero multiplicities created. The residual system is

$$
\mathcal{L}^{\prime}{ }_{\mathbb{P}}=\mathcal{L}_{2, \frac{a+b}{2}-\frac{5 m}{2}+18 \alpha}([a-m+4 \alpha, b-m+4 \alpha],[a-m+4 \alpha, b-m+4 \alpha])
$$

It is obvious that $\mathcal{L}_{\mathbb{P}}$ is empty if and only if $\mathcal{L}^{\prime}$ is empty.

We are now ready to prove the main result of this section.

Proof of Theorem 4.2 We want to prove that $\mathcal{L}=\mathcal{L}_{2,3 m+2 \alpha}\left((m+\alpha)^{2}, m^{8}\right)$ is empty for $m>9 \alpha$.

We assume by contradiction that there are some values of the parameters $a$ and $b$ for which both linear systems $\mathcal{L}_{\mathbb{P}}$ and $\mathcal{L}_{\mathbb{F}}$ are non-empty in the central fibre of the degeneration. If $\mathcal{L}_{\mathbb{P}}$ is non-empty, then the degree of $\mathcal{L}^{\prime} \mathbb{P}$ is positive. In particular

$$
a+b \geq 5 m-36 \alpha .
$$

On the other hand, since $\mathcal{L}_{\mathbb{F}}$ is non-empty, by Remark 4.8, we must have

$$
a+b \leq m .
$$

These two inequalities lead to a contradiction, and hence, the linear system $\mathcal{L}_{2,3 m+2 \alpha}((m+$ $\alpha)^{2}, m^{8}$ ) is empty.

In particular, Theorem 4.2 gives the following consequence.

Proposition 4.9 If $m \geq 8$, then the linear system $\mathcal{L}_{2,3 m+2}\left((m+1)^{2}, m^{8}\right)$ is empty.

Proof First, we check cases $m=8,9$ by computer. For $m \geq 10$, we apply Theorem 4.2 with $\alpha=1$.

\subsection{Classification of homogeneous linear systems $\mathcal{L}_{3,2 m+1}\left(m^{9}\right)$}

In this section, we completely classify homogeneous linear systems in $\mathbb{P}^{3}$ of degree $2 m+1$ with nine points. We start with the following lemma, which is an easy consequence of Proposition 4.9.

Lemma 4.10 The linear system $\mathcal{L}_{2,3 m+2}\left((m+1)^{2}, m^{8}\right)$ satisfies:

$$
\operatorname{dim}\left(\mathcal{L}_{2,3 m+2}\left((m+1)^{2}, m^{8}\right)\right)=\chi\left(\mathcal{L}_{\mathbb{P}^{1} \times \mathbb{P}^{1},(2 m+1,2 m+1)}\left(m^{9}\right)\right)
$$

for $m \leq 8$ and it is empty for $m \geq 8$. 
Proof We check by computer the statement for $m \leq 7$. For $m \geq 8$, we use Proposition 4.9.

By Lemma 2.1, the previous lemma has the following straightforward consequence.

Corollary 4.11 The linear system $\mathcal{L}_{\mathbb{P}^{1} \times \mathbb{P}^{1},(2 m+1,2 m+1)}\left(m^{9}\right)$ is non-special for every $m \geq 1$, and it is empty for $m \geq 8$.

We are ready now to prove the following classification result:

Theorem 4.12 A linear system $\mathcal{L}=\mathcal{L}_{3,2 m+1}\left(m^{9}\right)$ is special if and only if $m \geq 9$. In particular, we have:

- $\operatorname{dim}\left(\mathcal{L}_{3,2 m+1}\left(m^{9}\right)\right)=\operatorname{vdim}\left(\mathcal{L}_{3,2 m+1}\left(m^{9}\right)\right)$ for $m \leq 8$;

- $\operatorname{dim}\left(\mathcal{L}_{3,2 m+1}\left(m^{9}\right)\right)=60$ for $m \geq 7$;

- the quadric $Q$ through the nine base points is in the base locus of $\mathcal{L}$ with multiplicity $m-7$, for any $m \geq 8$.

Proof The restriction exact sequence (4.1) gives in this case:

$$
0 \rightarrow \mathcal{L}_{3,2(m-1)+1}\left((m-1)^{9}\right) \rightarrow \mathcal{L}_{3,2 m+1}\left(m^{9}\right) \rightarrow \mathcal{L}_{\mathbb{P}^{1} \times \mathbb{P}^{1},(2 m+1,2 m+1)}\left(m^{9}\right) \rightarrow 0 .
$$

We use induction on $m \geq 1$. The case $m=1$ is obvious. By Corollary 4.11 , we deduce that the linear system is non-special if and only if $m \leq 8$ (notice that if $m=8$, we have $\left.\chi\left(\mathcal{L}_{\mathbb{P}^{1} \times \mathbb{P}^{1},(17,17)}\left(8^{9}\right)\right)=0\right)$. Moreover, again from Corollary 4.11 , it follows that $\operatorname{dim}\left(\mathcal{L}_{3,2 m+1}\left(m^{9}\right)\right)=\operatorname{dim}\left(\mathcal{L}_{3,15}\left(7^{9}\right)=60\right.$ for all $m \geq 7$.

In order to prove that, for $m \geq 8$, the quadric is contained in the base locus of $\mathcal{L}$ with multiplicity $m-7$, it is enough to use Corollary 4.11 and to notice that $\operatorname{dim}\left(\mathcal{L}_{3,15}\left(7^{9}\right)\right) \neq$ $\operatorname{dim}\left(\mathcal{L}_{3,13}\left(6^{9}\right)\right)$.

A straightforward consequence of Theorem 4.12 is the following:

Corollary 4.13 Conjecture 5.1 holds for any homogeneous linear system with nine points of multiplicity $m$ and degree $d \leq 2 m+1$.

\section{Proof of Laface-Ugaglia Conjecture for linear systems with 9 points and multiplicities bounded by 8}

Let $\mathcal{L}=\mathcal{L}_{3, d}\left(m_{1}, \ldots, m_{9}\right)$ be the linear system of degree $d$ hypersurfaces of $\mathbb{P}^{3}$ with nine general multiple points of multiplicities $m_{1}, \ldots, m_{9}$. In this section, we will assume that $d \geq m_{1} \geq m_{2} \geq \cdots \geq m_{9}$. Let $Q=\mathcal{L}_{3,2}\left(1^{9}\right)$ be the unique quadric surface through the nine base points. We adopt the following notation

$$
q(\mathcal{L})=\chi\left(\mathcal{L}_{\mid Q}\right)=(d+1)^{2}-\sum_{i=1}^{9}\left(\begin{array}{c}
m_{i}+1 \\
2
\end{array}\right) .
$$

Laface and Ugaglia formulated their Conjecture in [27, Conjecture 4.1] and [29, Conjecture 6.3]. Following the definition of linear speciality introduced in [5], we can reformulate this Conjecture in the following way.

Conjecture 5.1 (Laface-Ugaglia Conjecture) Given a Cremona reduced linear system $\mathcal{L}$ in $\mathbb{P}^{3}$, we have 
(1) if $q(\mathcal{L}) \leq 0$, then $\operatorname{dim}(\mathcal{L})=\operatorname{dim}(\mathcal{L}-Q)$;

(2) if $q(\mathcal{L})>0$, then $\mathcal{L}$ is linearly non-special.

Remark 5.2 Since $\mathcal{L}$ is Cremona reduced, i.e. $m_{1}+m_{2}+m_{3}+m_{4} \leq 2 d$, it does not contain any plane in the base locus. Hence, Conjecture 5.1 says that if $q(\mathcal{L})>0$, then $\mathcal{L}$ is special if and only if $m_{1}+m_{2}-d \geq 2$ and in this case:

$$
\operatorname{dim}(\mathcal{L})=\operatorname{ldim}(\mathcal{L})=\chi(\mathcal{L})+\sum_{i, j}\left(\begin{array}{c}
m_{i}+m_{j}-d+1 \\
3
\end{array}\right),
$$

where ldim denotes the affine linear dimension, see [18, Definition 1.2].

Remark 5.3 If $q(\mathcal{L}) \leq 0$ and $\operatorname{dim}(\mathcal{L})=\operatorname{dim}(\mathcal{L}-Q)$, from the exact sequence (4.1), we obtain that

$$
\mathrm{h}^{1}(\mathcal{L})=\mathrm{h}^{1}(\mathcal{L}-Q)-q(\mathcal{L}) .
$$

This means that the quadric $Q$ is a special effect surface for the linear system $\mathcal{L}$.

Remark 5.4 We point out that a quadric surface in the base locus can give speciality even if it is contained with multiplicity one. Consider, for instance, the linear system $\mathcal{L}=\mathcal{L}_{3,8}\left(4^{7}, 3^{2}\right)$ for which $\operatorname{dim}(\mathcal{L})=6, \mathrm{~h}^{1}=-q(\mathcal{L})=1$. This system contains in its base locus the quadric $Q$ through the nine points, but does not contain $2 Q$.

This behaviour is different from the case of linear special effect varieties, for which any linear cycle of dimension $l$ contributes to the speciality only if its multiplicity in the base locus is at least $l+1$.

Remark 5.5 Notice that when a linear system $\mathcal{L}$ has a quadric surface as special effect variety, computing $\mathrm{h}^{1}(\mathcal{L})$ is quite difficult in general. In fact the quasi-homogeneous systems classified in Sect. 3 form a very special family for which we understand completely the situation, but this is not the case in general.

Let $\mathcal{L}$ be a linear system with $q(\mathcal{L}) \leq 0$. Assume there exists $\bar{k}$ such that

- $q(\mathcal{L}-k Q) \leq 0$ for any $0 \leq k \leq \bar{k}$ and $q(\mathcal{L}-(\bar{k}+1) Q)>0$,

- $\mathcal{L}-k Q$ restricts to non-special linear systems on the quadric $Q$, for any $0 \leq k \leq \bar{k}$.

Then, by using Remark 5.3, we get the following formula:

$$
\mathrm{h}^{1}(\mathcal{L})=-\sum_{k=0}^{\bar{k}} q(\mathcal{L}-k Q) .
$$

By using (5.2) in the case of quasi-homogeneous systems $\mathcal{L}_{3,2 m}\left(m^{8}, a\right)$ and using Remark 3.2, we recover exactly the formula $\mathrm{h}^{1}(\mathcal{L})=\left(\begin{array}{c}a+1 \\ 3\end{array}\right)+\left(\begin{array}{c}a \\ 2\end{array}\right)$ of Theorem 3.1. In this case, $\bar{k}=a$.

The problem in general is to determine the value of $\bar{k}$. Let us see an example: if $\mathcal{L}=$ $\mathcal{L}_{3,13}\left(8,6^{8}\right)$, then $q(\mathcal{L})=-8, q(\mathcal{L}-q)=-4, q(\mathcal{L}-2 Q)=-1$, while $q(\mathcal{L}-3 Q)=1>0$, and hence, we have $\mathrm{h}^{1}(\mathcal{L})=8+4+1=13$, and in this case, $\bar{k}=2$.

Remark 5.6 Given two vectors $v=\left(m_{1}, \ldots, m_{s}\right)$ and $v^{\prime}=\left(m_{1}^{\prime}, \ldots, m_{s}^{\prime}\right)$ in $\mathbb{N}^{s}$, we write $v^{\prime} \leq v$ if and only if $m_{i}^{\prime} \leq m_{i}$ for any $1 \leq i \leq s$.

It is easy to see that if a linear system $\mathcal{L}_{n, d}(v)$ is non-special and non-empty, then also any linear system $\mathcal{L}_{n, d}\left(v^{\prime}\right)$ is non-special and non-empty for any vector $v^{\prime} \leq v$. 
Now we establish Laface-Ugaglia Conjecture for any linear system with nine points of multiplicities bounded by 8 . We start with a lemma whose proof is essentially computational.

Lemma 5.7 If a linear system $\mathcal{L}=\mathcal{L}_{3, d}\left(m_{1}, \ldots, m_{9}\right)$ is such that $m=\max \left(m_{i}\right) \leq 8$ and $d<2 m$, then it satisfies Conjecture 5.1.

Proof First of all it is clear that if $d<m$, the system is empty, so we assume $d \geq m$. Assume that $\mathcal{L}$ is Cremona reduced, that is

$$
m_{1}+m_{2}+m_{3}+m_{4} \leq 2 d .
$$

Now if $d=m$, then by (5.3), we have that $m_{1}=m$ and $m_{2}<m$. Therefore by applying [5, Theorem 5.3], we have that if $\sum_{i=1}^{9} m_{i} \leq 3 d+2$, then $\mathcal{L}$ is linearly non-special. Hence, we can also assume

$$
\sum_{i=1}^{9} m_{i}>3 d+2 .
$$

For any $m \leq 8$, only the following systems satisfy conditions (5.3) and (5.4): $\mathcal{L}_{3,6}\left(6,2^{8}\right)$ and $\mathcal{L}_{3,7}\left(7,3,2^{7}\right)$. It is easy to check that these two systems are linearly non-special.

Assume now that $d \geq m+1$. We know, by [2], that Laface-Ugaglia Conjecture is true for any linear system with multiplicities bounded by 5 . So we can assume $6 \leq m \leq 8$.

Moreover, by applying again [5, Theorem 5.3], we have that if $\sum_{i=1}^{9} m_{i} \leq 3 d+3$, then $\mathcal{L}$ is linearly non-special. Hence, we can also assume

$$
\sum_{i=1}^{9} m_{i}>3 d+3 .
$$

Now we list all the possible linear systems which satisfy conditions (5.3) and (5.5), for any $5 \leq m \leq 8$ and any $m+1 \leq d \leq 2 m-1$. Then we prove that all the cases in the list satisfy the Conjecture using the following procedure. For any degree, we start to check the cases $\mathcal{L}=\mathcal{L}_{3, d}\left(m_{1}, \ldots, m_{8}\right)=\mathcal{L}_{3, d}(v)$ for the largest vectors $v$. We compute $\operatorname{dim}(\mathcal{L})$ by means of the computer system Macaulay2 as explained in Sect. 2.4.

If $\mathcal{L}$ is non-special and non-empty, then by Remark 5.6, also the linear system $\mathcal{L}_{3, d}\left(v^{\prime}\right)$ is non-special and non-empty, for any vector $v^{\prime} \leq v \in \mathbb{Z}^{9}$, and hence, we greatly reduce the number of cases to be checked.

If $\mathcal{L}$ is linearly non-special, then we apply [5, Lemma 5.5 and Remark 5.6] and [9, Theorem 1.2] and we obtain again that any system $\mathcal{L}_{3, d}\left(v^{\prime}\right)$, for $v^{\prime} \leq v \in \mathbb{Z}^{9}$, is linearly non-special. Hence, we further reduce the number of cases to be checked and we obtain at the end the lists contained in Tables 1, 2 and 3. Notice that in the tables, the special and linearly nonspecial systems are marked with $*$. By applying this procedure, we complete the proof of the lemma.

We give now the main result of this section:

Theorem 5.8 Conjecture 5.1 is true for any linear system $\mathcal{L}_{3, d}\left(m_{1}, \ldots, m_{9}\right)$ such that $m=$ $\max \left(m_{i}\right) \leq 8$.

Proof If the degree $d \leq 2 m-1$, the result follows from Lemma 5.7.

If $d=2 m$, by Theorem 3.1, we know that the quasi-homogeneous linear systems $\mathcal{L}_{3,2 m}\left(m^{8}, a\right)$ are special if and only if $2 \leq a \leq m$ and they satisfy Conjecture 5.1. Arguing as in Lemma 5.7, in order to complete the proof we need to check all linear systems satisfying (5.3) and (5.5) for any $6 \leq m \leq 8$. 
The list of these cases (reduced by Remark 5.6) is contained in Table 4, and we checked all of them by computer.

Now if $d \geq 2 m+1$, by Theorem 4.12 the linear system $\mathcal{L}_{3,2 m+1}\left(m^{9}\right)$ is non-special and non-empty. Hence, any homogeneous linear system $\mathcal{L}_{3, d}\left(m^{9}\right)$ for $d \geq 2 m+1$ is also non-special and non-empty.

Finally, we deduce that any (non-homogeneous) linear system $\mathcal{L}_{3, d}\left(m_{1}, \ldots, m_{9}\right)$ with $m_{i} \leq m$ is non-special and non-empty, by Remark 5.6. This completes the proof.

\subsection{Future directions}

We conclude this paper by pointing out possible future directions (both theoretical and computational) in establishing Laface-Ugaglia Conjecture for nine points. On the one hand, one can introduce further degenerations of $\mathbb{P}^{2}$ in order to obtain a better bound in the base locus lemma, Theorem 4.1. On the other hand, the combination of the results of Sect. 4 and of similar computer-based computations as the one performed in this section could improve the bound on the multiplicities of Theorem 5.8.

\subsection{Tables}

The linear systems marked with $*$ in Tables 1, 2 and 3 are linearly non-special, namely their dimension equals the linear expected dimension. All other linear systems have the quadric surface through nine points as special effect component, namely it splits off the system and gives speciality.

Table 1 The case $m=6$

\begin{tabular}{llrrr}
\hline Degree & $\left(m_{1}, m_{2}, m_{3}, m_{4}, m_{5}, m_{6}, m_{7}, m_{8}, m_{9}\right)$ & $q$ & $\mathrm{~h}^{0}$ & $\mathrm{~h}^{1}$ \\
\hline 11 & $(6,5,5,5,5,5,5,5,5)$ & 3 & 28 & 0 \\
11 & $(6,6,5,5,5,5,5,5,4)$ & 2 & 22 & 0 \\
11 & $(6,6,5,5,5,5,5,5,5)$ & -3 & 10 & 3 \\
11 & $(6,6,6,4,4,4,4,4,4)$ & 21 & 76 & 0 \\
10 & $(6,5,5,4,4,4,4,4,4)$ & 10 & 40 & 0 \\
10 & $*(6,6,4,4,4,4,4,4,4)$ & 9 & 35 & 1 \\
10 & $*(6,6,5,3,3,3,3,3,3)$ & 28 & 80 & 1 \\
9 & $(6,4,4,4,4,4,4,4,3)$ & 3 & 14 & 0 \\
9 & $(6,4,4,4,4,4,4,4,4)$ & -1 & 5 & 1 \\
9 & $*(6,5,4,3,3,3,3,3,3)$ & 18 & 50 & 1 \\
9 & $*(6,6,3,3,3,3,3,3,3)$ & 16 & 42 & 4 \\
8 & $*(6,4,3,3,3,3,3,3,3)$ & 8 & 20 & 1 \\
\hline
\end{tabular}


Table 2 The case $m=7$

\begin{tabular}{|c|c|c|c|c|}
\hline Degree & $\left(m_{1}, m_{2}, m_{3}, m_{4}, m_{5}, m_{6}, m_{7}, m_{8}, m_{9}\right)$ & $q$ & $\mathrm{~h}^{0}$ & $\mathrm{~h}^{1}$ \\
\hline 13 & $(7,6,6,6,6,6,6,6,6)$ & 0 & 28 & 0 \\
\hline 13 & $(7,7,6,6,6,6,6,5,5)$ & 5 & 42 & 0 \\
\hline 13 & $(7,7,6,6,6,6,6,6,4)$ & 4 & 36 & 0 \\
\hline 13 & $(7,7,6,6,6,6,6,6,5)$ & -1 & 22 & 1 \\
\hline 13 & $(7,7,6,6,6,6,6,6,6)$ & -7 & 10 & 10 \\
\hline 13 & $(7,7,7,5,5,5,5,5,5)$ & 22 & 98 & 0 \\
\hline 12 & $(7,6,6,5,5,5,5,5,5)$ & 9 & 49 & 0 \\
\hline 12 & $*(7,7,5,5,5,5,5,5,5)$ & 8 & 43 & 1 \\
\hline 12 & $*(7,7,6,4,4,4,4,4,4)$ & 32 & 112 & 1 \\
\hline 11 & $(7,5,5,5,5,5,5,5,4)$ & 1 & 15 & 0 \\
\hline 11 & $(7,5,5,5,5,5,5,5,5)$ & -4 & 5 & 5 \\
\hline 11 & $*(7,6,5,4,4,4,4,4,4)$ & 20 & 70 & 1 \\
\hline 11 & $*(7,6,6,3,3,3,3,3,3)$ & 38 & 110 & 2 \\
\hline 11 & $*(7,7,4,4,4,4,4,4,4)$ & 18 & 60 & 4 \\
\hline 11 & $*(7,7,5,3,3,3,3,3,3)$ & 37 & 105 & 4 \\
\hline 10 & $*(7,5,4,4,4,4,4,4,4)$ & 8 & 28 & 1 \\
\hline 10 & $*(7,5,5,3,3,3,3,3,3)$ & 27 & 74 & 2 \\
\hline 10 & $*(7,6,4,3,3,3,3,3,3)$ & 26 & 70 & 4 \\
\hline 10 & $*(7,7,3,3,3,3,3,3,3)$ & 23 & 58 & 10 \\
\hline 9 & $*(7,4,4,3,3,3,3,3,3)$ & 16 & 38 & 2 \\
\hline 9 & $*(7,5,3,3,3,3,3,3,3)$ & 15 & 35 & 4 \\
\hline 8 & $*(7,3,3,3,3,3,3,3,3)$ & 5 & 9 & 8 \\
\hline
\end{tabular}


Table 3 The case $m=8$

\begin{tabular}{|c|c|c|c|c|}
\hline Degree & $\left(m_{1}, m_{2}, m_{3}, m_{4}, m_{5}, m_{6}, m_{7}, m_{8}, m_{9}\right)$ & $q$ & $\mathrm{~h}^{0}$ & $\mathrm{~h}^{1}$ \\
\hline 15 & $(8,7,7,7,7,7,7,7,6)$ & 3 & 52 & 0 \\
\hline 15 & $(8,7,7,7,7,7,7,7,7)$ & -4 & 28 & 4 \\
\hline 15 & $(8,8,7,7,7,7,7,6,6)$ & 2 & 44 & 0 \\
\hline 15 & $(8,8,7,7,7,7,7,7,5)$ & 1 & 37 & 0 \\
\hline 15 & $(8,8,7,7,7,7,7,7,6)$ & -5 & 22 & 6 \\
\hline 15 & $(8,8,7,7,7,7,7,7,7)$ & -12 & 10 & 22 \\
\hline 15 & $(8,8,8,6,6,6,6,6,6)$ & 22 & 120 & 0 \\
\hline 14 & $(8,7,7,6,6,6,6,6,6)$ & 7 & 56 & 0 \\
\hline 14 & $*(8,8,6,6,6,6,6,6,6)$ & 6 & 49 & 1 \\
\hline 14 & $*(8,8,7,5,5,5,5,5,5)$ & 35 & 147 & 1 \\
\hline 14 & $*(8,8,8,4,4,4,4,4,4)$ & 57 & 203 & 3 \\
\hline 13 & $(8,6,6,6,6,6,6,5,5)$ & 4 & 34 & 0 \\
\hline 13 & $(8,6,6,6,6,6,6,6,4)$ & 3 & 28 & 0 \\
\hline 13 & $(8,6,6,6,6,6,6,6,5)$ & -2 & 15 & 2 \\
\hline 13 & $(8,6,6,6,6,6,6,6,6)$ & -8 & 5 & 13 \\
\hline 13 & $*(8,7,6,5,5,5,5,5,5)$ & 21 & 91 & 1 \\
\hline 13 & $*(8,7,7,4,4,4,4,4,4)$ & 44 & 154 & 2 \\
\hline 13 & $*(8,8,5,5,5,5,5,5,5)$ & 19 & 79 & 4 \\
\hline 13 & $*(8,8,6,4,4,4,4,4,4)$ & 43 & 148 & 4 \\
\hline 12 & $*(8,6,5,5,5,5,5,5,5)$ & 7 & 35 & 1 \\
\hline 12 & $*(8,6,6,4,4,4,4,4,4)$ & 31 & 105 & 2 \\
\hline 12 & $*(8,7,5,4,4,4,4,4,4)$ & 30 & 100 & 4 \\
\hline 12 & $*(8,8,4,4,4,4,4,4,4)$ & 27 & 85 & 10 \\
\hline 11 & $*(8,5,5,4,4,4,4,4,4)$ & 18 & 56 & 2 \\
\hline 11 & $*(8,6,4,4,4,4,4,4,4)$ & 17 & 52 & 4 \\
\hline 11 & $*(8,6,5,3,3,3,3,3,3)$ & 36 & 98 & 5 \\
\hline 11 & $*(8,7,4,3,3,3,3,3,3)$ & 34 & 90 & 10 \\
\hline 11 & $*(8,8,3,3,3,3,3,3,3)$ & 30 & 74 & 20 \\
\hline 10 & $*(8,4,4,4,4,4,4,4,4)$ & 5 & 14 & 8 \\
\hline 10 & $*(8,5,4,3,3,3,3,3,3)$ & 24 & 56 & 5 \\
\hline 10 & $*(8,6,3,3,3,3,3,3,3)$ & 22 & 50 & 10 \\
\hline 9 & $*(8,4,3,3,3,3,3,3,3)$ & 12 & 21 & 11 \\
\hline
\end{tabular}


Table 4 The case $d=2 m$

\begin{tabular}{|c|c|c|c|}
\hline$\left(m_{1}, m_{2}, m_{3}, m_{4}, m_{5}, m_{6}, m_{7}, m_{8}, m_{9}\right)$ & $q$ & $\mathrm{~h}^{0}$ & $\mathrm{~h}^{1}$ \\
\hline$(6,6,6,6,6,5,5,5,5)$ & 4 & 35 & 0 \\
\hline$(6,6,6,6,6,6,5,5,4)$ & 3 & 29 & 0 \\
\hline$(6,6,6,6,6,6,5,5,5)$ & -2 & 16 & 2 \\
\hline$(6,6,6,6,6,6,6,4,4)$ & 2 & 23 & 0 \\
\hline$(6,6,6,6,6,6,6,5,3)$ & 1 & 18 & 0 \\
\hline$(6,6,6,6,6,6,6,5,4)$ & -3 & 11 & 3 \\
\hline$(6,6,6,6,6,6,6,5,5)$ & -8 & 6 & 13 \\
\hline$(7,7,7,7,7,6,6,6,6)$ & 1 & 36 & 0 \\
\hline$(7,7,7,7,7,7,6,6,5)$ & 0 & 29 & 0 \\
\hline$(7,7,7,7,7,7,6,6,6)$ & -6 & 16 & 8 \\
\hline$(7,7,7,7,7,7,7,5,4)$ & 4 & 37 & 0 \\
\hline$(7,7,7,7,7,7,7,5,5)$ & -1 & 23 & 1 \\
\hline$(7,7,7,7,7,7,7,6,3)$ & 2 & 26 & 0 \\
\hline$(7,7,7,7,7,7,7,6,4)$ & -2 & 18 & 2 \\
\hline$(7,7,7,7,7,7,7,6,5)$ & -7 & 1 & 10 \\
\hline$(7,7,7,7,7,7,7,6,6)$ & -13 & 6 & 26 \\
\hline$(8,8,8,8,7,7,7,7,7)$ & 5 & 69 & 0 \\
\hline$(8,8,8,8,8,7,7,7,6)$ & 4 & 61 & 0 \\
\hline$(8,8,8,8,8,7,7,7,7)$ & -3 & 36 & 3 \\
\hline$(8,8,8,8,8,8,7,6,6)$ & 3 & 53 & 0 \\
\hline$(8,8,8,8,8,8,7,7,5)$ & 2 & 46 & 0 \\
\hline$(8,8,8,8,8,8,7,7,6)$ & -4 & 29 & 4 \\
\hline$(8,8,8,8,8,8,7,7,7)$ & -11 & 16 & 19 \\
\hline$(8,8,8,8,8,8,8,6,5)$ & 1 & 38 & 0 \\
\hline$(8,8,8,8,8,8,8,6,6)$ & -5 & 23 & 6 \\
\hline$(8,8,8,8,8,8,8,7,3)$ & 3 & 35 & 0 \\
\hline$(8,8,8,8,8,8,8,7,4)$ & -1 & 26 & 1 \\
\hline$(8,8,8,8,8,8,8,7,5)$ & -6 & 18 & 8 \\
\hline$(8,8,8,8,8,8,8,7,6)$ & -12 & 11 & 22 \\
\hline$(8,8,8,8,8,8,8,7,7)$ & -19 & 6 & 45 \\
\hline
\end{tabular}

Acknowledgments The authors would like to thank the referee for his/her many useful comments.

\section{References}

1. Alexander, J., Hirschowitz, A.: Polynomial interpolation in several variables. J. Algebraic Geom. 4(2), 201-222 (1995)

2. Ballico, E., Brambilla, M.C., Caruso, F., Sala, M.: Postulation of general quintuple fat point schemes in $\mathbb{P}^{3}$. J. Algebra 363, 113-139 (2012)

3. Bocci, C.: Special effect varieties in higher dimension. Collect. Math. 56(3), 299-326 (2005). ISSN: 0010-0757

4. Bocci, C.: Special effect varieties and (-1)-curves. Rocky Mt. J. Math. 40(2), 397-419 (2010)

5. Brambilla, M.C., Dumitrescu, O., Postinghel, E.: On a notion of speciality of linear systems in $\mathbb{P}^{n}$. Trans. Am. Math. Soc. 367, 5447-5473 (2015) 
6. Brambilla, M.C., Dumitrescu, O., Postinghel, E.: On the effective cone of $\mathbb{P}^{n}$ blown-up at $n+3$ points, arXiv:1501.04094 (2015)

7. Brambilla, M.C., Ottaviani, G.: On the Alexander-Hirschowitz theorem. J. Pure Appl. Algebra 212(5), 1229-1251 (2008)

8. Catalisano, M.V., Geramita, A.V., Gimigliano, A.: Higher secant varieties of Segre-Veronese varieties. In: Projective varieties with unexpected properties, pp. 81-107 (2005)

9. Chandler, K.: The geometric interpretation of Fröberg-Iarrobino conjectures on infinitesimal neighbourhoods of points in projective space. J. Algebra 286(2), 421-455 (2005)

10. Ciliberto, C., Dumitrescu, O., Miranda, R., Roé, J.: Emptiness of homogeneous linear systems with ten general base points. In: Classification of algebraic varieties, EMS Ser. Congr. Rep., Eur. Math. Soc., Zürich, pp. 189-195 (2011)

11. Ciliberto, C., Miranda, R.: Degenerations of planar linear systems. J. Reine Angew. Math. 501, 191-220 (1998)

12. Ciliberto, C., Miranda, R.: Linear systems of plane curves with base points of equal multiplicity. Trans. Am. Math. Soc. 352, 4037-4050 (2000)

13. Ciliberto, C., Miranda, R.: Homogeneous interpolation on ten points. J. Algebraic Geom. 20, 685-726 (2011)

14. Ciliberto, C.: Geometrical aspects of polynomial interpolation in more variables and of Waring's problem. In: European Congress of Mathematics, vol. I (Barcelona, 2000), pp. 289-316, Progr. Math., 201, Birkhäuser, Basel, (2001)

15. De Volder, C., Laface, A.: On linear systems of $\mathbb{P}^{3}$ through multiple points. J. Algebra 310(1), 207-217 (2007)

16. Dolgachev, I.: Weyl groups and Cremona transformations, Singularities, Part 1 (Arcata, Calif., 1981), pp. 283-294, Proc. Sympos. Pure Math., 40, Amer. Math. Soc., Providence, RI (1983)

17. Dumitrescu, O.: Plane curves with prescribed triple points: a toric approach. Commun. Algebra 41(5), 1626-1635 (2013)

18. Dumitrescu, O., Postinghel, E.: Vanishing theorems for linearly obstructed divisors, arXiv:1403.6852 (2014)

19. Gimigliano, A.: On linear systems of plane curves, Ph.D. Thesis, Queen's University, Canada (1987)

20. Giuffrida, S., Maggioni, R., Ragusa, A.: On the postulation of 0-dimensional subschemes on a smooth quadric. Pac. J. Math. 155(2), 251-282 (1992)

21. Guardo, E., Van Tuyl, A.: Fat points in $\mathbb{P}^{1} \times \mathbb{P}^{1}$ and their Hilbert functions. Can. J. Math. 56(4), 716-741 (2004)

22. Grayson, D., Stillman, M.: Macaulay 2, a software system for research in algebraic geometry. http://www. math.uiuc.edu/Macaulay2/

23. Harbourne, B.: The geometry of rational surfaces and Hilbert functions of points in the plane. Can. Math. Soc. Conf. Proc. 6, 95-111 (1986)

24. Hirschowitz, A.: Une conjecture pour la cohomologie des diviseurs sur les surfaces rationnelles génériques. J. Reine Angew. Math. 397, 208-213 (1989)

25. Laface, A.: On linear systems of curves on rational scrolls. Geom. Dedicata 90, 127-144 (2002)

26. Laface, A., Postinghel, E.: Secant varieties of Segre-Veronese embeddings of $\left(\mathbb{P}^{1}\right)^{r}$. Math. Ann. 356(4), 1455-1470 (2013)

27. Laface, A., Ugaglia, L.: On a class of special linear systems on $\mathbb{P}^{3}$. Trans. Am. Math. Soc. 358(12), 5485-5500 (2006). (electronic)

28. Laface, A., Ugaglia, L.: On multiples of divisors associated to Veronese embeddings with defective secant variety. Bull. Belg. Math. Soc. Simon Stevin 16(5), 933-942 (2009)

29. Laface, A., Ugaglia, L.: Standard classes on the blow-up of $\mathbb{P}^{n}$ at points in very general position. Commun. Algebra 40, 2115-2129 (2012)

30. Postinghel, E.: A new proof of the Alexander-Hirschowitz interpolation theorem. Ann. Mat. Pura Appl. 191(1), 77-94 (2012)

31. Ran, Z.: Enumerative geometry of singular plane curves. Invent. Math. 97, 447-465 (1989)

32. Segre, B.: Alcune questioni su insiemi finiti di punti in geometria algebrica. Atti Convegno Intern. di Geom. Alg. di Torino 15-33 (1961)

33. Van Tuyl, A.: An appendix to a paper of M. V. Catalisano, A. V. Geramita and A. Gimigliano. The Hilbert function of generic sets of 2-fat points in $\mathbb{P}^{1} \times \mathbb{P}^{1}$ : Higher secant varieties of Segre-Veronese varieties. In: Projective varieties with unexpected properties, pp. 109-112 (2005) 